\title{
Caracterización de métodos de evaluación de desempeño para equipos de desarrollo de software
}

\author{
Characterization of performance evaluation methods \\ for software development teams \\ José Julián Zapata Arbeláez ${ }^{1}$ \\ Bell Manrique-Losada ${ }^{1 *}$ \\ Gloria Piedad Gasca-Hurtado ${ }^{1}$ \\ Liliana Machuca-Villegas ${ }^{1,2}$ \\ Recibido 5 de agosto de 2020, aceptado 16 de octubre de 2020 \\ Received: August 5, 2020 Accepted: October 16, 2020
}

\begin{abstract}
RESUMEN
La evaluación de desempeño pretende medir el grado con el que un empleado se ajusta y cumple con el perfil deseado. Los resultados de esta medición permiten generar recomendaciones, buscando mejorar el desempeño de las funciones por parte del empleado. Además, los resultados favorecen la gestión del talento humano organizacional, relacionada con: crecimiento profesional, bonificaciones, compensaciones, y selección de candidatos.

En este artículo se presenta un proceso de análisis, comparación y caracterización de métodos de evaluación de desempeño. Se ha realizado este proceso con el propósito de aprovechar los beneficios de la medición del desempeño e identificar aquellos métodos más empleados en equipos de desarrollo de software. Este análisis permitió caracterizarlos e identificar los factores que determinan su uso, así como los criterios que permiten especificar y diferenciar su aplicación en la industria de software.

Se estableció una metodología compuesta por tres fases para llevar a cabo el propósito de este trabajo. A partir de esta metodología se logró conducir la caracterización de los métodos de evaluación de desempeño para equipos de desarrollo de software.

Se identificaron 11 métodos de evaluación de desempeño convencionales y 10 aproximaciones a evaluación de desempeño para equipos de desarrollo de software. Se incluyeron los métodos convencionales usados por aquellas propuestas enfocadas a evaluación de desempeño de equipos de desarrollo de software. A partir de factores de caracterización y comparación definidos, se consigue una caracterización, donde se destaca la evaluación de competencias técnicas, por encima de las genéricas, psicológicas o de experiencia.
\end{abstract}

Palabras clave: Evaluación por competencias, métodos de evaluación, evaluación de desempeño, gestión de talento humano, desarrollo de software.

\section{ABSTRACT}

The purpose of the performance evaluation is to measure the degree in which an employee of an organization adjusts or complies with the desired profile. The results of this measurement allow generating recommendations to improve the performance of the functions by the employee.

Moreover, the results favor the human talent management, related to professional growth, bonuses, compensations, and candidate selection.

\footnotetext{
1 Universidad de Medellín. Facultad de Ingeniería. Medellín, Colombia.

E-mail: jjzapata81@hotmail.com; bmanrique@udem.edu.co; gpgasca@udem.edu.co

2 Universidad del Valle. Facultad de Ingeniería. Cali, Colombia. E-mail: liliana.machuca@correounivalle.edu.co

* Autor de correspondencia: bmanrique@udem.edu.co
} 
In this paper, an analysis, comparing, and characterization process of the performance evaluation methods are presented. This process was realized to take advantage of the benefices of performance evaluation and identify which methods of performance evaluation are most used in software development teams. This analysis allows us to characterize these methods and identify the factors that determine their usage, as well as the criteria that allow them to specify and differentiate their application in the software industry. A methodology is integrated into three phases to carry out the purpose of this work. Through this methodology, it was possible to conduct the characterization of performance evaluation methods for software development teams.

We identified 11 conventional performance evaluation methods and 10 approaches to performance evaluation for software development teams. We included conventional methods because the particular proposals for software development teams use some of them. From this set of studies, the analysis of characterization and comparison factors was established.

This characterization shows that the evaluation of technical competences takes precedence, over generic, psychological, or experience competences.

Keywords: Competences assessment, assessment methods, performance evaluation, human talent management, software development.

\section{INTRODUCCIÓN}

Desde mediados de los años 80, se empezó a producir un cambio de cara al papel que juega el empleado dentro de una organización [1], dicho cambio logró que se dejara de considerar a la persona, como un gasto y comenzara a considerarse el principal recurso de una empresa [2]. Otro elemento importante que empezó a ocurrir en ese momento, fue la concepción del término 'competencia', que hace referencia a la forma en la que un empleado debe cumplir sus funciones. Estos cambios han llevado a que en la gestión de recursos humanos sea necesario incorporar métodos y técnicas para medir las competencias, proceso que se conoce hoy en día como evaluación de desempeño.

La evaluación de desempeño es la principal herramienta que tiene una organización para realizar una buena administración del talento humano que posee [3] y esta se refiere a los métodos y procesos que puede realizar la empresa para determinar el nivel de desempeño con el que los empleados cumplen sus funciones. Con los resultados obtenidos de una evaluación de desempeño, la organización puede establecer programas, tales como: planeación estratégica, compensación salarial, promociones, distribución de carga laboral, planes de formación, selección de personal y proyección de una imagen objetiva e imparcial hacia sus empleados. De esta manera es posible agrupar las motivaciones para realizar una evaluación de desempeño en razones gerenciales, desarrollo profesional y formación [4].

Por lo general, el proceso de evaluación de desempeño incluye la medición y la retroalimentación del empleado. Sin embargo, la evaluación tiende a tener cierto nivel de rechazo por parte de los evaluados [5] debido a factores como: i) inexistencia de una medida perfecta, dado que los sistemas de medición pueden ser confusos y difíciles de estandarizar; uso de escalas erróneas de evaluación, puesto que están sujetas a la experiencia de quien diseña la evaluación y a la subjetividad del evaluador, entre otros; ii) desde el punto de vista del evaluador, el proceso está altamente ligado a su nivel de experiencia y conocimiento, pero además se puede ver afectado por elementos de índole personal como la relación que tiene con el evaluado o, incluso, el estado anímico al momento de realizar la evaluación; y iii) desde el punto de vista del evaluado, el resultado del proceso de evaluación puede considerarse bajo en relación al trabajo que desempeña o comparado con el resultado de otros compañeros; o simplemente atribuir un mal resultado a factores meramente externos, desconociendo sus propios aspectos de mejora.

Diferentes estudios plantean la manera de aplicar técnicas que mitiguen los factores antes mencionados $[6,7]$, para que los empleados puedan percibir la evaluación como una herramienta más justa y objetiva, con todos los beneficios que esto conlleva $[8,9]$. 
En el contexto de la industria del desarrollo de software se han aplicado también implementación de métodos o técnicas desde la evaluación de desempeño convencional, integrando además en la evaluación, competencias técnicas y genéricas. Además, diversos trabajos han realizado acercamientos hacia el análisis cuantitativo [6] desde valores cualitativos obtenidos durante el proceso evaluativo, como por ejemplo la escala de Likert [10], la cual ha tenido variaciones en sus alternativas de implementación $[11,12]$ y en la forma de ponderar o valorar las respuestas [13].

En el presente artículo se presenta el proceso de identificación de los métodos de evaluación de desempeño más empleados en equipos de desarrollo de software, su caracterización en términos de los factores que determinan su uso y los criterios que permiten especificar y diferenciar su aplicación en la industria de software. El objetivo de la identificación y caracterización de los métodos de evaluación es consolidar un conjunto de criterios y factores que faciliten a las organizaciones de desarrollo de software, valorar los métodos de evaluación de la productividad utilizados en sus equipos de desarrollo. Con esta valoración se pretende ofrecer a las organizaciones desarrolladoras de software, un conjunto de técnicas de medición adaptadas al contexto, es decir al desarrollo de software. Además, esta caracterización es un primer paso hacia una metodología de medición de productividad en equipos de desarrollo de software, como parte de un trabajo de investigación para la Maestría en Ingeniería de Software de la Universidad de Medellín.
El resto del trabajo se estructura de la siguiente forma: en la siguiente sección se presenta la metodología de revisión donde se muestran las fases y tareas que la componen. Después, se presenta la caracterización de métodos de evaluación de desempeño, donde se incluyen aproximaciones a la evaluación de desempeño para equipos de desarrollo de software como un background de este trabajo e insumo para el análisis y la comparación de dichas aproximaciones. Finalmente, en la última sección se muestran las conclusiones de este trabajo.

\section{METODOLOGÍA DE REVISIÓN}

La metodología que se llevó a cabo en esta investigación está compuesta por tres fases. Cada una de estas fases está descrita en términos de tareas. En la Figura 1 se muestra la composición de la metodología que conduce a la revisión propuesta en este trabajo. Las dos primeras fases de la metodología se han propuesto a la luz de las recomendaciones que comprenden la Revisión Sistemática de Literatura [14]. Parte de los resultados de estas fases han sido consolidados en el apartado de aproximaciones a la evaluación de desempeño, constituido como background de este trabajo. La tercera fase se establece a partir de tareas que facilitaron el análisis de los métodos de evaluación del desempeño identificados. En dicha fase se incluye el análisis de factores de comparación que fueron utilizados para establecer la caracterización de las aproximaciones identificadas. A continuación, se explican los detalles de cada una de las fases de la metodología de revisión definida.

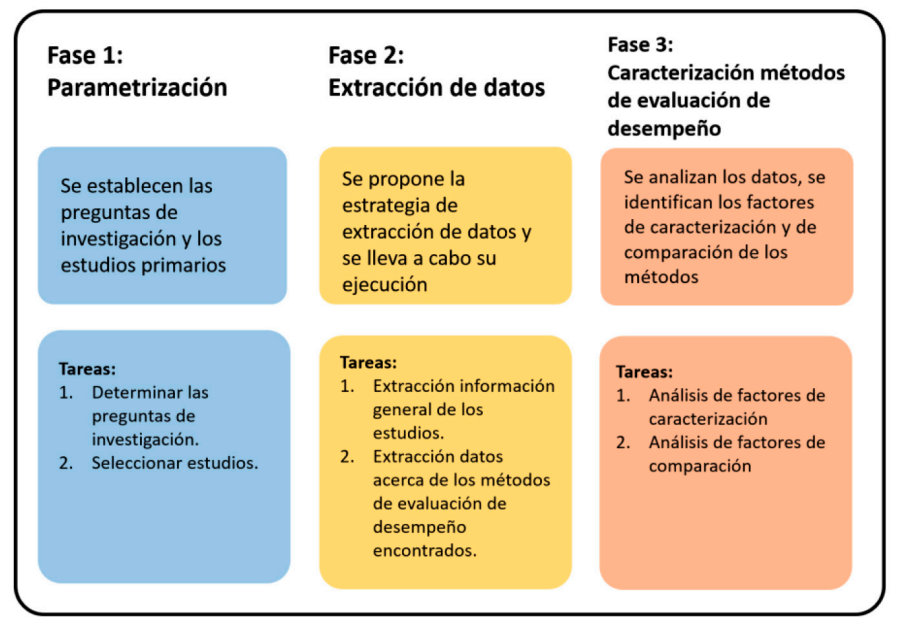

Figura 1. Metodología de revisión. 


\section{Fase 1. Parametrización}

El objetivo de esta fase es establecer los parámetros de partida y la necesidad del estudio de los métodos de evaluación del desempeño en equipos de desarrollo de software. Para lograr definir estos parámetros de partida, se siguen las recomendaciones del Protocolo de Revisión Sistemática de Literatura [14] y se inicia la Fase 1 con la definición de preguntas de investigación. El punto de partida para definir estas preguntas es el objetivo general de trabajo de grado de maestría que da origen a este artículo.

Tarea 1. Determinar las preguntas de investigación. Las preguntas de investigación que orientan el desarrollo de esta revisión son:

- ¿Cuáles son los métodos más usados para realizar la evaluación de desempeño en los empleados?

- ¿Qué factores permiten caracterizar los métodos identificados?

- ¿Qué criterios de comparación se pueden establecer para analizar los métodos identificados?

Tarea 2. Seleccionar estudios. Para la selección inicial de estudios, se creó una cadena de búsqueda a partir de los diferentes objetivos y preguntas de investigación planteados como parte de los parámetros de partida de este trabajo. Los términos que se incluyen en esta cadena de búsqueda están alineados con las palabras clave de la investigación y las preguntas definidas en la Tarea 1. El resultado es el siguiente:

employee AND performance AND (evaluation OR appraisal) AND (methods OR techniques) AND (advantage OR disadvantage) AND (software development)

En la Tabla 1 se listan las bases de datos seleccionadas para la consulta y la cantidad de artículos encontrados inicialmente.

Para continuar con el filtrado de los resultados preliminares y, finalmente, llegar a los estudios primarios, se enuncian los siguientes criterios de inclusión (CI) y de exclusión (CE):

- CI1. Trabajos cuyo objeto principal de estudio sean los diferentes métodos de evaluación de desempeño.

- CI2. Trabajos que desde su resumen e introducción aborden, mencionen la evaluación de desempeño como problemática principal.

- CI3. Libros y artículos en español y en inglés.

- CI4. Libros y artículos que mencionen de forma explícita, ventajas o desventajas de métodos de evaluación de desempeño.

- CE1. Trabajos cuyos métodos propuestos no estén aplicados a la industria de desarrollo de software.

De todo el proceso de selección se obtienen 41 artículos que constituyen la muestra de estudios a ser analizados en esta investigación.

\section{Fase 2. Extracción de Datos}

El objetivo de esta fase es extraer los datos de acuerdo a los parámetros de interés definidos a partir de las preguntas de investigación establecidas en la Fase 1.

Se ha extraído información general de los estudios en función de autores, país, palabras clave, método de evaluación y resumen. También se ha extraído información de acuerdo con los factores de caracterización y los criterios de comparación. Para el caso de la caracterización se han establecido los siguientes atributos a extraer de los estudios: objetivo, modelo, métrica, herramienta y tipo de competencias a evaluar. Para el caso de la comparación se han definido los siguientes criterios: costos, subjetividad, tamaño del equipo/ organización, enfoque técnico, enfoque personal, complejidad, efectos en la motivación y toma de decisiones/mejora organizacional.

En la siguiente sección se describen los resultados obtenidos en la fase 3 de esta metodología.

Tabla 1. Síntesis de los resultados.

\begin{tabular}{|c|c|r|r|r|r|r|}
\hline Motor & \multicolumn{1}{c|}{ i } & \multicolumn{1}{c|}{ ii } & iii & iv & $\mathbf{v}$ & Total \\
\hline A & 2.436 & 608 & 1.877 & 134 & 52 & 5.107 \\
\hline B & 15 & 3 & 12 & 8 & 3 & 41 \\
\hline
\end{tabular}

i) Science Direct, ii) Scopus, iii) AMC, iv) Emerald, v) IEEE.

A) resultados iniciales de búsqueda, B) preselección basada en el resumen. 


\section{CARACTERIZACIÓN DE MÉTODOS DE EVALUACIÓN DE DESEMPEÑO}

A continuación, se presenta la caracterización de los métodos convencionales para la evaluación de desempeño. Esta caracterización de métodos convencionales se considera fundamental para estas propuestas utilizadas en el contexto de equipos de desarrollo de software.

\section{Métodos convencionales de evaluación de desempeño}

- Evaluación $360^{\circ}$. Este método consiste en valorar las competencias del empleado, obteniendo información desde las personas que están a su alrededor. Esta información puede provenir tanto de niveles múltiples dentro de la empresa (empleado, supervisor, compañeros), como de fuentes externas (clientes). En las evaluaciones $360^{\circ}$, se determinan los estilos y las necesidades de desarrollo directivo y se detectan los estilos de dirección dominantes y los poco desarrollados. Proporciona diferentes perspectivas (empleado, supervisor, compañeros) que genera mayor retroalimentación, aportando información de resultados y opiniones [1, 15]. Pese a ser uno de los métodos más utilizados, también es uno de los más controversiales, ya que la falta de seguimiento al momento posterior a la evaluación, hace que la retroalimentación generada no sea aprovechada en el futuro.

- Autoevaluación. Es un método donde el empleado se evalúa a sí mismo, por lo que no solo participa en el proceso de evaluación, sino que también puede establecer su propio proyecto de mejora y describir su puesto, logros personales, dificultades que ha tenido y cuestiones que podría mejorar [16].

- Dirección o administración por objetivos. El supervisor y el empleado establecen conjuntamente los objetivos de desempeño deseables y las metas. Lo ideal es que se establezcan por acuerdo mutuo y que sean mesurables de manera objetiva. Cada empleado tiene unas metas específicas fijadas por él mismo, pero dentro de un esquema general, supervisado por su jefe. La descripción de la meta se acompaña de una descripción detallada de cómo hará ese empleado para alcanzarla [17].

- Registro de incidentes críticos. El evaluador registra las conductas clave que deciden la consecución eficaz de los objetivos del puesto, es decir, las conductas que han demostrado ser clave para el desempeño eficiente en el trabajo. Proporciona una gran retroalimentación al empleado y reduce el efecto de distorsión que tienen en la memoria los acontecimientos recientes ya que abarca todo el periodo evaluado [1].

- Escala de clasificación conductual. Se presenta una escala en la que aparecen características a evaluar y el evaluador indica hasta qué grado el empleado posee cada una de ellas comparando el desempeño del empleado con unos parámetros preestablecidos. Se suelen asignar valores numéricos a cada aspecto evaluado [18].

- Escalas Mixtas. Es una modificación del método anterior, donde en lugar de evaluar las características con una escala se le proporcionan al evaluador descripciones más específicas de cada característica [18].

- Listas de verificación. La persona que otorga la calificación selecciona oraciones de la lista que describen el desempeño y las características del empleado. Se asignan puntuaciones a las listas (normalmente cada uno de los aspectos contenidos en las listas de verificación tienen puntuaciones diferentes) [19].

- Distribución forzosa. Cada evaluador ubica a sus empleados en diferentes clasificaciones, habiendo un número máximo de empleados por cada categoría [20].

- Simulación y ejercicios de carácter práctico. Consiste en someter a prueba a los sujetos evaluados planteándoles situaciones o casos prácticos similares, en términos de competencias exigidas, a los que se viven en el puesto de trabajo. Así pueden demostrar el nivel de competencia que poseen. Este procedimiento se recomienda especialmente cuando no es posible la observación en entornos reales, esto puede ocurrir cuando la evaluación se realiza antes de la incorporación a un puesto de trabajo, enmarcada en procesos de selección de personas, o simplemente, porque la presencia de evaluadores en el entorno laboral no lo permite. Normalmente se combina con los otros métodos, para recolectar evidencias sobre las competencias demostradas por el personal evaluado [1].

- Escala convencional o Jerarquización simple o múltiple. El evaluador clasifica a los empleados que dependen de él en una relación jerarquizada, ordenando a los evaluados de mejor a peor en relación a su aportación global a las tareas de 
su departamento, clasificando a los empleados por orden de eficiencia. En la jerarquización múltiple se emiten tantas listas como sub-criterios sean analizados y permite destacar aquellos empleados que son considerados superiores, para motivarlos al valorar su trabajo. También proporciona a los empleados considerados inferiores la posibilidad de mejorar dándoles un ejemplo de cuál es el ideal de conducta esperado por la empresa [18].

- Comparaciones por pares. El evaluador debe comparar al evaluado con todos los que pertenecen al mismo grupo (misma ocupación o mismas características), eligiendo para cada par el empleado con nivel de desempeño superior para el parámetro objeto de la evaluación. El empleado cuyo desempeño sea considerado superior mayor número de veces es el mejor en el parámetro elegido [21].

\section{Aproximaciones a evaluación de desempeño para equipos de desarrollo de software}

La caracterización de los métodos identificados permite conducir a la selección de propuestas representativas para el desempeño de equipos de desarrollo de software. Por lo tanto, en este apartado se muestra un background de las propuestas más representativas para evaluar desempeño de equipos de desarrollo de software. Estas aproximaciones están seleccionadas a partir del contexto de la industria del desarrollo de software, donde se ha identificado que toma más importancia la evaluación de competencias técnicas, que la evaluación de competencias genéricas, psicológicas o de experiencia.

Ampuero y Baldoquín [22] proponen un sistema de soporte a la decisión para apoyar la asignación de personal a proyectos de desarrollo de software, a partir de roles especificados previamente. Aplican el Modelo formal de asignación (MARHEPS) en el diseño del sistema que apoya el proceso de selección de roles y asignación a proyectos.

De Carvalho [23] define el sistema informático RHPlan para la asignación de personal a proyectos de software, también a partir de la evaluación de competencias genéricas, técnicas y psicológicas. Esta propuesta se centra en la asignación individual de personas a tareas o roles del proyecto, pero no consideran aspectos de formación del equipo en general. Este sistema es a su vez usado como base en la especificación de un proceso de gestión de recursos humanos llamado MR-MPS, desarrollado por Morais [24].

Amâncio et al. [25] proponen un modelo de procesos para la gestión de talento humano en pequeñas casas de software. Esta propuesta está basada en la guía PMBok, siguiendo el flujo de trabajo del proceso definido en la metodología RUP. El modelo propuesto incluye el gerenciamiento de las fases por las que debe pasar un proyecto de desarrollo: planeación de recursos humanos, contratación y movilización del equipo del proyecto, desarrollo del equipo del proyecto y gerenciamiento. La evaluación de desempeño se ejecuta como un sub-proceso dentro de la fase de desarrollo del equipo, siguiendo técnicas de cuestionarios e indicadores, así como observación directa.

Serres [26] presenta un Sistema para evaluación de desempeño (SED) para empresas de desarrollo de software, que busca conocer si el desempeño de los empleados es adecuado, o si por el contrario existen deficiencias que deben corregirse, y considerarse para el diseño de planes de mejora. Asimismo, busca contribuir a la mejora continua de los empleados y al registro de incidentes críticos que se hayan producido a lo largo del periodo de evaluación. La autora define categorías de evaluación, que incluyen la evaluación de actitudes (compromiso, cooperación, responsabilidad, etc.), aspectos relativos a la conducta del empleado, y aspectos asociados a competencias genéricas de desempeño (iniciativa, creatividad, liderazgo, orientación al cliente, etc.). Se definen intervalos y puntuaciones mínimas por categorías para evaluar el rendimiento de los empleados.

Bhatnagar [27] propone una estrategia de gestión de talento para evaluar el nivel de compromiso de los empleados de compañías TI. Esta estrategia se fundamenta en un estudio estadístico centrado en encuestas que identifica características claves de la actuación de un empleado en tres factores: (i) cultura organizacional, asociada con comunicación interna, centralidad en el cliente y cultura de trabajo; (ii) Planificación profesional e incentivos, asociado con aspectos de reconocimiento personal, oportunidad de crecimiento, compensaciones y promoción; (iii) apoyo organizacional, asociado con infraestructura de soporte, claridad del rol desempeñado, entrenamiento, entre otros. 
Delta Technology \& Management Services [28] diseñan una metodología para evaluación de empleados y programa de desarrollo de equipos. Como base definen tipos de medida asociados a los factores de adecuación, importancia y precisión, con métricas financieras, de resultados, influenciables, reacciones de pares y tiempo relacionado. Definen como etapas para el diseño del sistema de evaluación, las siguientes: diagnóstico, procedimientos, diseño, implementación piloto e introducción.

Fernandes y otros [29] presentan el Sistema SAN (Stochastic Automata Networks) para modelar y analizar equipos de desarrollo de software y así predecir su desempeño en diferentes escenarios. A partir de la definición de unas asignaciones a proyectos y la ejecución de eventos, el sistema genera una estimación de dedicación de empleados a dichos eventos y a su vez provee probables índices de desempeño. Finalmente, el impacto de la productividad de todo el equipo de desarrollo se predice.

Cantú-Mata y otros [30] proponen un modelo estructural para evaluar las competencias del desarrollador de software. El modelo se centra en la evaluación de la calidad del servicio del desarrollador, principalmente orientado hacia medir o evaluar la calidad de los servicios de Outsourcing de software. Los elementos a medir están asociados a la 'calidad del servicio' y a competencias 'genéricas' (ej. Rendición de cuentas, solución de conflictos, iniciativa, planificación) y 'técnicas' (actividades asociadas a las actividades de desarrollo: diseño, codificación, pruebas, configuración, etc.) del desarrollador de software.

Rey y otros [31] definen un sistema experto para Evaluación de Competencias de los candidatos a un cargo específico en el campo del desarrollo de software. Esta propuesta pretende apoyar la selección de personal y su incorporación o asignación del ya efectivo a labores concretas de desarrollo de software. El sistema se centra en las competencias genéricas (ej. Instrumentales, interpersonales, sistemáticas) de los roles de desarrollador (administrador del proyecto, analista, diseñador, programador) y en las competencias técnicas que permiten la ejecución de tareas específicas del puesto de trabajo.

Sanyal y otros [32], definen la evaluación de desempeño como factor estratégico, tanto como para atraer como para retener talento humano, teniendo en cuenta la importancia del rol del recurso humano dentro de una organización. Métodos como la evaluación 360, pueden ser muy bien aprovechados, gracias a la retroalimentación que proporcionan, para establecer procesos de crecimiento profesional, tener esquemas de compensación salarial, crear planes de mentoría y planear actividades de capacitación en áreas específicas que ayuden a mejorar la calidad a la hora de crear líneas de código. En el trabajo se proponen cuatro elementos claves dentro de la evaluación de desempeño: i) establecer metas u objetivos, relacionados directamente con competencias de índole técnico; ii) monitoreo constante; iii) evaluación y, finalmente iv) retroalimentación de los resultados obtenidos en todo el proceso.

Maghsoodi y otros [33], resaltan la importancia de la evaluación de desempeño dentro del área de administración de talento humano, cuyo rol es cada vez más significativo por la ventaja competitiva que logra en una organización. Los autores de este trabajo consideran como factor crítico de éxito de la evaluación de desempeño, los aspectos relacionados con la precisión de los métodos de evaluación. Por lo anterior, en este trabajo se propone un enfoque denominado MULTIMOORA para definir un sistema de evaluación de desempeño que genere información precisa y segura para la toma de decisiones de la organización.

Esen y otros en [34], mencionan la importancia de la evaluación de desempeño para determinar las habilidades que posee un empleado dentro de una organización, resaltando especialmente la alineación de las habilidades con los objetivos de la empresa. En este trabajo los autores proponen una evaluación de desempeño utilizando variables lingüísticas y lógica difusa, con el fin de minimizar los posibles elementos de subjetividad. El modelo planteado se presenta como una buena alternativa para ser utilizado en diferentes contextos laborales.

Un análisis de las aproximaciones a evaluación de desempeño para equipos de desarrollo de software estudiadas hasta aquí, permite clasificarlas y seleccionar aquellas que conformarán la caracterización de las aproximaciones, objetivo de este estudio. A continuación, se muestra el resultado del análisis que facilitó establecer dicha caracterización. 


\section{Caracterización de las aproximaciones identificadas}

En la Tabla 2 se analizan y sintetizan aspectos de las propuestas descritas en la sección anterior, asociados a los siguientes factores:

(i) enfoque del modelo que fundamenta cada método.

(ii) herramienta computacional que permite su implementación en las empresas.

(iii) métrica asociada (si la tiene).

(iv) tipo de competencia que incluye, ya sea técnica, genérica, psicológica o asociada a la experiencia del empleado.

Las empresas de desarrollo de software enfrentan diversos retos en la gestión de sus equipos humanos, por lo cual resulta vital que se logren implementar procesos de gestión de recursos y evaluación de desempeño, basados en el enfoque por competencias. El proceso de evaluación de desempeño de empleados de equipos de desarrollo de software, por hacer parte de la categoría de talento humano TI, merece atención especial, más aún cuando en general, se lleva a cabo de forma empírica. Cada día más es necesario contar con herramientas y métodos que apoyen a los directivos a enfrentar este proceso, dado que es evidente el incremento en la demanda de roles y empleados a considerar.

Análisis y comparación de las aproximaciones En la Tabla 3 se presenta una comparación de las propuestas de evaluación de desempeño para

Tabla 2. Análisis factores por métodos.

\begin{tabular}{|c|c|c|c|c|c|c|c|}
\hline \multirow{2}{*}{$\begin{array}{l}\text { Método/ } \\
\text { Factores }\end{array}$} & \multirow{2}{*}{ Modelo asociado } & \multirow{2}{*}{ Métrica } & \multirow{2}{*}{ Herramienta } & \multicolumn{4}{|c|}{ Tipo de competencias a evaluar } \\
\hline & & & & Técnicas & Genéricas & Psicológicas & Experiencia \\
\hline $\begin{array}{l}\text { Ampuero y } \\
\text { Baldoquín, } \\
\text { (2010) [22] }\end{array}$ & $\begin{array}{l}\text { Modelo formal de asig- } \\
\text { nación (MARHEPS), a } \\
\text { partir de factores para } \\
\text { asignar roles de un } \\
\text { proyecto. }\end{array}$ & $\begin{array}{l}\text { Métricas de tiempo, } \\
\text { tamaño y defectos. }\end{array}$ & TEAMSOFT + & SI & SI & SI & SI \\
\hline $\begin{array}{l}\text { De Carvalho, } \\
(2003)[23]\end{array}$ & Modelo de procesos. & N/A & RHPlan & SI & SI & SI & NO \\
\hline \begin{tabular}{|l|} 
Morais, \\
$(2009)[24]$
\end{tabular} & MR-MPS. & N/A & N/A & SI & NO & NO & SI \\
\hline $\begin{array}{l}\text { Amâncio et al., } \\
\text { (2009) [25] }\end{array}$ & Modelo de procesos. & $\begin{array}{l}\text { Progreso vs entregas. } \\
\text { Casos de éxito. Tasa de } \\
\text { rotación del personal. }\end{array}$ & N/A & SI & SI & NO & SI \\
\hline $\begin{array}{l}\text { Serres, } \\
\text { (2014) [26] }\end{array}$ & $\begin{array}{l}\text { Sistema para evaluación } \\
\text { de desempeño. }\end{array}$ & $\begin{array}{l}\text { Contribución al logro } \\
\text { de objetivos. }\end{array}$ & \begin{tabular}{|l|} 
SED (sistema \\
evaluación de \\
desempeño). \\
\end{tabular} & SI & SI & SI & SI \\
\hline $\begin{array}{l}\text { Bhatnagar, } \\
\text { (2007) [27] }\end{array}$ & $\begin{array}{l}\text { Estrategia para evaluar el } \\
\text { nivel de compromiso de } \\
\text { empleados TI. }\end{array}$ & N/A & N/A & - & SI & SI & - \\
\hline $\begin{array}{l}\text { Delta } \\
\text { Technology, } \\
\text { (2011) [28] }\end{array}$ & $\begin{array}{l}\text { Metodología para eva- } \\
\text { luación de empleados. }\end{array}$ & \begin{tabular}{|l|} 
Financieras, de resulta- \\
dos, reacciones de pares \\
y tiempo relacionado, \\
(adecuación, importan- \\
cia y precisión).
\end{tabular} & N/A & SI & SI & - & - \\
\hline $\begin{array}{l}\text { Fernandes et al., } \\
\text { (2011) [29] }\end{array}$ & $\begin{array}{l}\text { Modelo para predicción } \\
\text { de desempeño de equipos. }\end{array}$ & $\begin{array}{l}\text { Disponibilidad } \\
\text { Calidad de soporte } \\
\text { Dedicación. }\end{array}$ & $\begin{array}{l}\text { Sistemas SAN } \\
\text { y PEPS. }\end{array}$ & SI & SI & - & SI \\
\hline $\begin{array}{l}\text { Cantu-Mata } \\
\text { et al., } \\
\text { (2014) [30] }\end{array}$ & $\begin{array}{l}\text { Modelo de evaluación de } \\
\text { competencias del desarro- } \\
\text { llador de software. } \\
\end{array}$ & N/A & N/A & SI & SI & NO & SI \\
\hline $\begin{array}{l}\text { Rey et al., } \\
\text { (2013) [31] }\end{array}$ & $\begin{array}{l}\text { Sistema para selección de } \\
\text { personal desarrollador de } \\
\text { software. }\end{array}$ & $\begin{array}{l}\text { Métricas técnicas aso- } \\
\text { ciados a cada rol, como: } \\
\text { abstracción y lógica en } \\
\text { el programador. }\end{array}$ & $\begin{array}{l}\text { Prototipo } \\
\text { de sistema } \\
\text { experto. }\end{array}$ & SI & SI & NO & NO \\
\hline
\end{tabular}


equipos de desarrollo de software. Para esta comparación se han definido criterios relacionados con el costo, la subjetividad, el tamaño del equipo/ organización, el enfoque técnico, el enfoque personal, la complejidad y su apoyo a la toma de decisiones/ mejora organizacional. El grado de cumplimiento de cada uno de estos factores se mide identificando su nivel bajo (0), medio (1) o alto (2) en el que la propuesta cumple con el criterio.

Respecto a la comparación descrita en la Tabla 3, se puede decir que los enfoques de evaluación propuestos presentan un nivel medio y alto respecto al costo que implica su implementación. Esto tiene que ver con la inversión en dinero, esfuerzo, tiempo y capacitación que debe hacerse. También se aprecia un nivel bajo y medio de subjetividad, debido a que algunos de los trabajos propuestos se fundamentan en modelos matemáticos, por lo tanto, se posibilita una evaluación más objetiva [22, 28, 29]. Desde la perspectiva del tamaño del equipo/organización se indica que los enfoques se orientan hacia medianas y grandes empresas de desarrollo de software, esto facilita tener un amplio alcance en el número de personas a evaluar. Para el caso del enfoque de la evaluación hacia competencias de tipo técnico o más personal, se observa que el enfoque técnico prima sobre el enfoque personal.

Analizando la complejidad de aplicación de las propuestas de evaluación se encontró que existe un grado de complejidad medio y alto en ellas. En este caso se requiere conocer bien el modelo propuesto y capacitar a las personas involucradas. Para el criterio relacionado con los efectos en la motivación del equipo de desarrollo de software, sólo algunos estudios expresan su influencia hacia este aspecto. Finalmente, todos los enfoques buscan apoyar la toma de decisiones en la gestión del equipo de trabajo de software. El propósito de evaluar el desempeño del equipo va encaminado hacia la mejora en procesos y bienestar de las personas.

Bajo este contexto de comparación, se percibe que los enfoques de evaluación estudiados son exigentes en la inversión de recursos a utilizarse, así como en la complejidad de su aplicación. Estos criterios pueden constituir una desventaja para la implementación de estos factores. Sin embargo, se destaca el grado de objetividad que los enfoques pretenden alcanzar a través de sus métodos de evaluación. Permitiendo con ello, que el empleado no se sienta amenazado por la evaluación y que se mida lo que realmente se debe medir. Esto también se aprecia en la tendencia hacia una evaluación orientada hacia aspectos técnicos.

\section{CONCLUSIONES}

En este trabajo se identificó un conjunto de métodos de evaluación de desempeño empleados en equipos

Tabla 3. Comparación de aproximaciones.

\begin{tabular}{|l|c|c|c|c|c|c|c|c|}
\hline \multirow{2}{*}{ Método } & \multicolumn{9}{|c|}{ Criterios de comparación } \\
\cline { 2 - 9 } & Costos & Subjetividad & $\begin{array}{c}\text { Tamaño } \\
\text { del equipo/ } \\
\text { organización }\end{array}$ & $\begin{array}{c}\text { Enfoque } \\
\text { técnico }\end{array}$ & $\begin{array}{c}\text { Enfoque } \\
\text { personal }\end{array}$ & Complejidad & $\begin{array}{c}\text { Efectos } \\
\text { en la } \\
\text { motivación }\end{array}$ & $\begin{array}{c}\text { Toma de } \\
\text { decisiones/mejora } \\
\text { organizacional }\end{array}$ \\
\hline $\begin{array}{l}\text { Ampuero y Baldoquín, } \\
\text { (2010) [22] }\end{array}$ & 2 & 0 & 2 & 2 & 2 & 1 & 0 & 2 \\
\hline De Carvalho, (2003) [23] & 1 & 1 & 1 & 1 & 2 & 0 & 1 & 2 \\
\hline Morais, (2009) [24] & 1 & 1 & 1 & 2 & 0 & 1 & 0 & 2 \\
\hline Amâncio et al., (2009) [25] & 1 & 1 & 1 & 2 & 0 & 1 & 2 & 2 \\
\hline Serres, (2014) [26] & 2 & 1 & 1 & 2 & 1 & 1 & 1 & 2 \\
\hline Bhatnagar, (2007) [27] & 2 & 1 & 1 & 1 & 2 & 1 & 2 & 2 \\
\hline Delta Technology (2011) [28] & 2 & 0 & 2 & 2 & 0 & 1 & 2 & 2 \\
\hline Fernandes et al., (2011) [29] & 2 & 0 & 1 & 2 & 1 & 2 & 0 & 2 \\
\hline Cantu-Mata et al., (2014) [30] & 2 & 1 & 1 & 2 & 1 & 0 & 0 & 2 \\
\hline Rey et al., (2013) [31] & 1 & 0 & 0 & 2 & 1 & 1 & 1 & 2 \\
\hline
\end{tabular}


de desarrollo de software. Cada uno de ellos se caracteriza en términos de factores que determinan su uso, así como los criterios que permiten especificar y diferenciar su aplicación en la industria de software.

Se identificaron estudios primarios en el contexto de esta investigación, de donde se extrajo información relevante de acuerdo con los factores de caracterización y los criterios de comparación. La caracterización se centró en los atributos: objetivo, modelo, métrica, herramienta y tipo de competencias a evaluar. Los criterios de comparación se definen en términos de costos, subjetividad, tamaño del equipo, enfoque técnico, enfoque personal, complejidad, y efectos en la motivación y/o toma de decisiones.

Se propusieron factores de caracterización de los métodos/técnicas de evaluación de desempeño en contextos de desarrollo de software, así como criterios que permiten analizar las ventajas y desventajas que pueden tener dichos métodos/técnicas en este tipo de industrias. En términos generales se percibe que en las aproximaciones analizadas se requiere niveles altos de inversión de recursos, dada la complejidad de su aplicación. Estos pueden constituir una desventaja para su implementación y la necesidad de proponer métodos y herramientas diferentes que flexibilicen su aplicación.

En el contexto de la industria de software, según las aproximaciones a evaluación de desempeño estudiadas, toma más importancia la evaluación de competencias técnicas, por encima de las genéricas, psicológicas o de experiencia. Bajo este resultado, es importante mantener en la evaluación de estas competencias un equilibrio que facilite una evaluación integral y equitativa debido a que el proceso de desarrollo de software se centra en las personas.

A pesar de que el número de estudios encontrados en este contexto es bajo, los métodos de evaluación orientados a la gestión de equipos de software descritos en este trabajo ofrecen a las organizaciones, nuevas alternativas para valorar y proponer técnicas de medición adecuadas a su entorno y de esta manera enfrentar los restos de la gestión de su talento humano.

La caracterización y comparación de los enfoques estudiados constituye una base teórica para el planteamiento de propuestas para la evaluación de desempeño de equipos de desarrollo de software. De esta manera, los resultados aquí obtenidos podrán ser tenidos en cuenta en el diseño de una metodología de evaluación del desempeño procurando tener un enfoque más estándar y cuantitativo.

\section{REFERENCIAS}

[1] J. Gil Flores. "La evaluación de competencias laborales”. Educación XX1, pp. 83-106. 2007.

[2] C. Obisi. "Employee performance appraisal and its implication for individual and organizational growth". Australian Journal of Business and Management Research. Vol. $1 \mathrm{~N}^{\circ}$ 9, pp. 92-97, 2011.

[3] D. Dina and M. Schodl. "Performance Appraisal and Evaluation". International Encyclopedia of the Social \& Behavioral Sciences. Vol. 17, pp. 716-721. 2015.

[4] S. Apaka, S. Gümüşb, G. Önerc and H.G. Gümüşd. "Performance appraisal and a field study". Procedia-Social and Behavioral Sciences. Vol. 229, pp. 104-114. 2016.

[5] L. Hui and G. Qin-xuan. "Performance appraisal: what's the matter with you?". The 6th International Conference on Mining Science \& Technology. Vol. $1 \mathrm{~N}^{\circ}$ 1, pp. 17511756. 2009.

[6] M. Valdés-Padrón, R. Garza-Ríos, I. PérezVergara, M. Gé-Varona y A.R. Chávez-Vivó. "Una propuesta para la evaluación del desempeño de los trabajadores apoyada en el uso de técnicas cuantitativas". Ingeniería Industrial. Vol. $36 \mathrm{~N}^{\circ}$ 1, pp. 48-57. 2015.

[7] A. Frederiksena, F. Lange and B. Kriechel. "Subjective performance evaluations and employee careers". Journal of Economic Behavior \& Organization. Vol. 134, pp. 408- 429. 2017.

[8] X. Islamia, E. Mulollia and N. Mustafab. "Using Management by Objectives as a performance appraisal tool for employee satisfaction". Future Business Journal. Vol. 4 $\mathrm{N}^{\mathrm{o}}$ 1, pp. 94-108. 2018.

[9] M.K. Sanyala and S.B. Biswasb. "Employee Motivation from Performance Appraisal Implications: Test of a theory in the Software Industry in West Bengal (India)". Procedia Economics and Finance. Vol. 11, pp. 182196. 2014. 
[10] R. Likert. "A technique for measurement of attitudes". Archives of Psychology, pp. 5-55. 1932.

[11] H.N. Boone and D.A. Boone. "Analyzing Likert Data". Journal of extension. Vol. 50 $\mathrm{N}^{\circ}$ 2, pp. 1-5. 2012.

[12] D.L. Clason and T.J. Dormody. "Analyzing data measured by individual Likert-type items". Journal of agricultural education. Vol. $35 \mathrm{~N}^{\circ}$ 4, p. 4.1994.

[13] M.G. Bozal. "Escala mixta Likert-Thurstone". Revista Andaluza de Ciencias Sociales, pp. 81-95. 2006.

[14] B. Kitchenham and S. Charters. "Guidelines for performing Systematic Literature reviews in Software Engineering". Technical report, Version 2.3. 2007. DOI: 10.1145/1134285.1134500.

[15] F. Pereira, S. Gutiérrez, L. Sardi y M. Villamil. "Las competencias laborales y su evaluación mediante el modelo 360 grados". Cuadernos Latinoamericanos de Administración. Vol. 4 $\mathrm{N}^{\circ}$ 6, pp. 69-105. 2008.

[16] C. Lusthaus, M.H. Adrien, G. Anderson y F. Carden. "Mejorando el desempeño de las organizaciones: método de autoevaluación". IDRC. 2001.

[17] X. Islamia, E. Mulollia and N. Mustafab. "Using Management by Objectives as a performance appraisal tool for employee satisfaction". Future Business Journal. Vol. 4 $\mathrm{N}^{\circ}$ 1, pp. 94-108. 2018.

[18] I. Chiavenato. "Administración de recursos humanos: El capital humano de las organizaciones". Mc Graw Hill. 2007.

[19] J.M. García. "La evaluación del desempeño. ¿Qué es, para qué sirve y cuáles son sus métodos? - Universidad de Málaga". Contribuciones a la Economía. 2010. URL: http:// www.eumed.net/ce/index.html

[20] A. Pérez Montejo. "Evaluación del Desempeño Laboral”. Gestión. Vol. 2 Nº 9. 2009.

[21] J. Osorio Gómez, J. Herrera Umaña, M. Vinasco y M. Adrián. "Modelo para la evaluación del desempeño de los proveedores utilizando AHP”. Ingeniería y Desarrollo. 2008.

[22] M. Ampuero y M.G. de la Peña. "Un sistema de soporte a la decisión para la asignación de recursos humanos a equipos de proyectos de software". Revista investigación operacional. Vol. 31 No $^{\circ}$, pp. 61-69. 2010.
[23] L.R.C. Schnaider. "Planejamento da alocação de recursos humanos em Ambientes de desenvolvimento de software orientados à Organização". Master Thesis. COPPE Universidade Federal do Rio de Janeiro. 2003.

[24] S.R.G. Morais. "Uma abordagem para a gerência de recursos humanos de organizações de software". Master Thesis. Universaidade de Fortaleza. Brazil. 2009.

[25] S.F. Amâncio, H. Augustus, X. Costa e V.V.D. Camargo. "Gerência de Recursos Humanos para uma Fábrica de Software de Pequeno Porte". In V Workshop Um Olhar Sóciotécnico Sobre a Engenharia de Software, pp. 86-95. 2009.

[26] M. Serres Peris. "Diseño de un sistema de evaluación del desempeño para una empresa de desarrollo de software de gestión empresarial". Tesis Universidad Politécnica de Valencia. España. 2014.

[27] J. Bhatnagar. "Talent Management strategy of employee engagement in Indian ITES employees: Key to retention". Employee Relations. Vol. 29 No 6, pp. 640-663. 2007.

[28] Delta Technology \& Management Services Pvt. Ltd. "Ways to Measure Your Developers Performance". 2011. URL: https://www. deltaintech.com/media/4674/ways $\% 20$ to $\% 20$ measure $\% 20$ your $\% 20$ developers $\% 20$ performancev2.pdf

[29] P. Fernandes, A. Sales, A.R. Santos and T. Webber. "Performance Evaluation of Software Development Teams: A Practical Case Study". Electronic Notes in Theoretical Computer Science. Vol. 275. 2011. DOI: https://doi. org/10.1016/j.entcs.2011.09.006.

[30] J. Cantú-Mata, J. Segoviano Hernández, R. Penilla-Leal y R. Zurita-Alarcón. "Modelo estructural para evaluar las competencias del desarrollador de software". Revista Facultad de Ingeniería, pp. 90-100. 2014. ISSN: 2422-2844.

[31] H. Rey, J. Ballesteros y A. Pérez. "Sistema experto para la selección de personal desarrollador de software". Ingenio Magno. Vol. $4 \mathrm{~N}^{\mathrm{o}}$ 1, pp. 75-81. 2013.

[32] M.K. Sanyal, S.B. Biswas and R. Ghosh. "Implication of Performance Appraisal process on Employee Engagement Mediated Through the Development and Innovation Oriented Culture: A Study on the Software 
Professionals of IT Companies in West Bengal". Proceedings of the First International Conference on Intelligent Computing and Communication, pp. 277-294. 2016.

[33] A.I. Maghsoodi, G. Abouhamzeh, M. Khalilzadeh and E.K. Zavadskas. "Ranking and selecting the best performance appraisal method using the MULTIMOORA approach integrated Shannon's entropy". Frontiers of Business Research in China, pp. 1-21. 2008.

[34] H. Esen, T. Hatipoğlu and A.İ. Boyac1. "A Fuzzy Approach for Performance Appraisal: The Evaluation of a Purchasing Specialist". Computational Intelligence, pp. 235-250. 2015. 